\title{
Hegel ou le possible réel. \\ La critique hégélienne des idéaux en question
}

\author{
Claire $_{\text {Agès }}$
}

Partant de l'analyse anthropologique des âges de la vie par Hegel dans l'Encyclopédie, nous nous proposons de présenter sa critique des idéaux. Hegel fustige chez le jeune homme sa tendance à opposer le réel et l'idéal et dégage les risques à la fois individuels et collectifs que cette vision du monde fait courir. De cette analyse, suit la conclusion qu'il n'y a pas de possible en dehors du réel, pas de substantialité hors du monde. Autrement dit, et contre la première impression de la plupart des hommes, possible et réel, devoir-être et être, s'identifient. Pourtant, si l'aversion du réel inspirée par l'attachement aux idéaux représente, selon Hegel, un danger, le procès du devoir-être par la philosophie spéculative n'est pas non plus sans risque. Ne signe-t-il pas, comme le dira Nietzsche, la défaite de la volonté face au poids des choses? On peut au contraire montrer, en lisant, dans la Doctrine de l'essence, le chapitre intitulé «L'effectivité » dans la section du même nom, que la reconnaissance de l'intimité du possible et de l'effectif, d'une part, produit en retour une conception plus riche, plus noble du possible - elle lui donne en somme des lettres de noblesse - et, d'autre part, conduit à y lire une affirmation de la contingence. Notre argumentaire vise à établir qu'en disant que l'effectif est possible et que le possible est effectif, Hegel ne les détruit pas mais leur confère au contraire leur détermination propre. 
Le paragraphe 396 de la Philosophie de l'esprit de l'Encyclopédie (édition de 1830) est consacré à la description du cours naturel des âges de la vie. Le jeune homme y est présenté comme l'esprit qui oppose une imagination, un devoir-être ou un espoir au monde présent, qu'il juge non conforme à ces idéaux. On trouve, dans l'addition au § 396, une description plus complète de la disposition d'esprit du jeune homme. Celui-ci chercherait satisfaction dans un universel substantiel et non plus, comme l'adolescent, dans un universel singulier, un homme. Pourtant, cet universel est encore subjectif, puisque le jeune homme vit d'un idéal, qui peut être un idéal d'amitié, mais qui a trait le plus souvent à un état rêvé du monde. Contrairement à l'enfant, il ne vit plus en paix avec le monde qui l'entoure. Bien au contraire, il nourrit une grande rancœur à l'égard d'un monde qu'il pense sans vérité, sorti de ses gonds, indigne de ce qui doit être. Mais le jeune homme ne se contente pas de s'opposer au monde présent. Il n'est pas inactif. La pensée de l'idéal lui donne aussi l'énergie nécessaire pour travailler à supprimer cette opposition, à réaliser l'idéal. Il entend ainsi transformer le monde et il se forme pour pouvoir réaliser ses idéaux. C'est ainsi, dit Hegel, qu'il devient un homme.

Pourtant, dans cette attitude, Hegel ne voit qu'apparence de noblesse et de désintéressement. S'il s'agit d'un passage obligé de la vie, celui qui conduit à l'âge adulte, il n'en faut pas moins détromper ceux qui verraient dans ce désintéressement de façade, dans cette exaltation, la vérité du cœur humain, le meilleur de l'homme. Hegel renverse tout d'abord l'idée que le jeune homme serait désintéressé, par opposition à l' « homme fait ${ }^{1}$ », qui ne se préoccuperait que de ses intérêts particuliers. Au contraire, le jeune homme reste occupé de ses vues subjectives et de son développement personnel, quand l'homme fait agit pour le monde et non simplement pour lui-même. De plus, le jeune homme se trompe lourdement sur le monde, quand il le juge privé de vérité. Il n'a pas compris, note Hegel, que 
ce qu'il y a de substantiel dans son idéal existe déjà dans le monde. Il n'a pas compris que «le monde comme un monde subsistant-par-soi » est «pour l'essentiel tout achevé » (Hegel, Encyclopédie III 438 [Add. § 396]).

Hegel ne traite pas cette erreur avec tendresse. Ces idéalistes-là ne lui inspirent aucune indulgence. Il y voit au contraire un grand danger. Le jeune homme, tout préoccupé qu'il est de ses idéaux, peut ressentir des difficultés à entrer dans la vie pratique, à s'occuper des autres, à traiter avec la singularité, à s'occuper des choses de la vie. Or, existe toujours le risque de ne pas réussir ce passage et de devenir malade de l'impossible réalisation immédiate de ses idéaux. L'homme risque alors de devenir hypocondriaque (hypochondrisch), lui qui ne parvient pas à se défaire de son aversion pour la réalité et qui continue à placer la vérité en lui-même dans la pensée subjective de l'idéal. Si, pour nous, l'hypocondrie désigne couramment chez quelqu'un une anxiété excessive et pathologique à propos de sa santé, du fonctionnement de ses organes, ou quelqu'un qui s'exagère ses souffrances, Hegel semble employer ce terme en un sens à la fois plus général et plus classique, pour désigner une humeur sombre, mélancolique et soucieuse (un atrabilaire). Mais l'intéresse surtout le fait que les hypocondriaques sont en somme des malades imaginaires : de même que ces malades se plaignent de maux inexistants, ou plus exactement de maux qui n'ont pas de fondement organique, de même les hommes qui s'entêtent, leur jeunesse passée, dans la poursuite de l'idéal, se plaignent du réel sans raison valable ${ }^{2}$. L'homme atteint d'hypocondrie a en outre la particularité de jouir souvent d'une apparence de bonne santé, quoiqu'il soit triste, et son affection semble chronique et de longue durée. Il n'est pas facile en effet, note Hegel, d'échapper à cette hypocondrie, quoique souvent on ne l'aperçoive pas, car elle a le pouvoir de se dissimuler. Le risque est de la voir s'étendre sur toute une vie, d'autant que les symptômes peuvent être très graves, puisque l'homme peut sombrer et devenir tout à fait incapable. 
Pour cause précise de cette hypocondrie, Hegel donne l'aversion de la réalité effective associée à la poursuite de l'idéal. Que fait donc le jeune homme et que continue de faire celui qui ne parvient pas à faire son deuil de cette posture de jeunesse? Il oppose idéal et réel, devoir-être et être, parce qu'il croit qu'il y a du possible hors du monde tel qu'il est : il croit à un autre monde possible. Il croit, par conséquent, à la distinction du possible et du réel, puisqu'il s'échine à démontrer qu'autre chose est possible que la réalité effective, que le monde présent. Il pense, en outre, que l'effectif peut être irrationnel et qu'il y a une rationalité de ce qui n'est pas effectif. L'homme fait, au contraire, comprendrait l'identité du possible et du réel, autrement dit l'effectivité de ce qui est rationnel. Celui-ci comprend que ce qu'il y a de substantiel dans l'idéal n'est pas simplement possible mais bien réel, autrement dit, que le possible est aussi réel, que le concept existe. Alors, il comprend que ce qu'il y a de proprement idéal — de non substantiel — dans l'idéal est vain.

Rester un jeune homme paraît alors non une aubaine ou une qualité, mais un danger. Pourtant, il semble que le plus grand nombre ait tendance à donner raison au jeune homme. En effet, celui-ci ne raisonne-t-il pas exactement de la même façon que la plupart des hommes, qui opposent le possible conçu et le réel, et qui font du possible un réel auquel ferait défaut l'existence? En opposant l'idéal au monde, le jeune homme ne se comporte-t-il pas en homme ordinaire, qui, distinguant possible et réel, pense qu'il existe des possibles subsistants par soi en dehors de la réalité? Affirmant que l'un de ces possibles non effectifs correspond à l'idéal, il exemplifierait alors une conception assez traditionnelle du rapport entre possible et réel. De cette conception que Hegel semble viser derrière l'opposition de l'idéal et du réel, on trouve parfois des traces chez Hegel lui-même. Ainsi, au début de l'Introduction des Leçons sur la philosophie de l'histoire, à propos du caractère encore abstrait et général du concept de l'esprit, il déclare : 
Le principe, comme la maxime, la loi est quelque chose d'intérieur, qui comme tel, quelque vrai qu'il soit en lui-même, n'est pas complètement réel. Des fins, des maximes, etc.... se trouvent dans notre pensée, d'abord dans nos intentions intérieures, mais pas encore dans la réalité. Ce qui est en soi une possibilité, un pouvoir, mais qui n'est pas parvenu encore de l'intérieur à l'existence. Pour la réalité, un second moment doit s'adjoindre, la mise en acte, la réalisation dont le principe est la volonté d'une façon générale, l'activité de l'homme. C'est par cette activité seule que ce concept, ainsi que les déterminations en soi, sont réalisées, produites, car prises immédiatement en elles-mêmes, elles sont sans valeur. (Hegel, Leçons 30)

Certes, Hegel paraît ici distinguer possible et réel, pour dénoncer l'absence de valeur de ce qui est simplement abstrait. Il peut sembler alors que le possible corresponde au réel moins son caractère effectif, et le réel au possible plus le caractère d'effectivité. D'une telle différence, il n'est pas impossible de conclure, comme le jeune homme, qu'il s'agit alors de choisir un possible qu'on juge digne et vrai et d'entreprendre de le faire entrer dans les faits. N'importe quel possible pourrait devenir réel, à condition de s'en donner les moyens. Hegel ne partage évidemment pas cette position, qu'il juge au contraire très risquée.

\section{II.}

Le $§ 396$ de l'Encyclopédie et son addition font du jeune homme l'homme qui trouve le monde présent inadapté à ses idéaux, quand l'homme fait serait l'homme qui reconnaîtrait le caractère nécessaire et rationnel du monde qui se trouve déjà là, tout achevé. La maturité conduirait à reconnaître qu'avoir des idéaux ne change pas le monde. Par conséquent, opposer le devoir-être à l'être est signe d'immaturité. Le désir de l'idéal a donc une temporalité propre : la jeunesse. Pourtant, Hegel indiquait aussi que cette erreur peut durer toute la vie et devenir une maladie. Il semble que l'exemple du jeune homme illustre une tendance humaine très générale qui serait simplement plus visible à cet âge de la vie.

Hegel ne dénonce pas autre chose dans l'Introduction des Leçons sur la philosophie de l'histoire, quand il s'en prend au caractère infondé du mécontentement des hommes. 
Certes, il précise que «la jeunesse est toujours mécontente», alors que «l'âge rend plus doux », mais il rencontre aussi ces plaintes chez des hommes qui ne sont plus tout jeunes : «Ce qui rend les hommes mécontents moralement (et c'est un mécontentement dont ils se font gloire), c'est qu'ils ne trouvent pas que le présent correspond à des fins qu'ils considèrent comme justes et bonnes (aujourd'hui en particulier les idéaux de constitutions); ils opposent à une telle condition le devoir selon eux de ce qui serait le droit en l'affaire » $(38)^{3}$.

Cette tendance à l'indignation face à la condition du monde semble un travers général de la nature humaine, quoique Hegel considère plus précisément son inflation chez ses contemporains et à son époque ${ }^{4}$, où il voit une lutte de positions cherchant chacune à justifier la nécessité de substituer à l'ordre du monde un ordre plus élevé. Il est ici extrêmement sévère à l'égard de tous ces mécontents, qui une fois fait le procès de leur temps, se plaignent que leurs idéaux ne se réalisent pas, et accusent encore le monde de ne pas laisser sa chance au droit. Eux aussi sont convaincus qu'autre chose que ce qui est, est possible, et ils débattent pour savoir quelle possibilité devrait remplacer l'ordre injuste. Néanmoins, Hegel n'est pas sans reconnaître une certaine rationalité à ce mécontentement. Il est vrai, en effet, que chez ces soupirants à l'idéal, c'est moins l'intérêt particulier ou la passion que la raison, le droit, la liberté, qui demandent satisfaction. Pourtant, là aussi, il traite sans ménagement ces hommes qui se plaignent du monde. Ceux-ci ont beau invoquer la raison comme fin dernière de leur entreprise et être pleins de bonnes intentions, leurs aspirations restent subjectives et strictement individuelles. Hegel n'y perçoit pas du désintéressement mais de la prétention, car chacun de ces hommes érige comme le plus juste et le plus haut ce qu'il s'imagine individuellement être tel. Il voit donc dans la défense de l'idéal — et dans celle d'un possible non-réel — un simple subjectivisme, la critique subjective et négative de celui qui généralise ce qui lui fait défaut. 
Ces mécontents commettent la même erreur que le jeune homme de l'Encyclopédie. Si le jeune homme pèche par immaturité, ces mécontents s'égarent en choisissant la facilité : ils ne saisissent pas quel est le fond véritable du réel et le jugent à la légère sans y avoir séjourné. Ils ne comprennent pas que la nature de leurs idéaux exclut qu'ils se réalisent (fantaisie), que le fond des choses est rationnel et qu'il n'y a pas de possible hors du réel. La position hégélienne est sans appel : la philosophie doit s'opposer à de telles démarches, en expliquant que le non-réel ne peut être tenu pour un possible permettant de juger du réel, mais qu'il faut reconnaître qu'il «n'a qu'une existence factice ».

\section{III.}

De ces deux analyses suit la même conclusion : qu'il n'y a pas de possible en dehors du réel, pas de substantialité hors du monde. Autrement dit, et contre la première impression de la plupart des hommes, possible et réel, devoir-être et être, s'identifient. L'idéal et le devoir-être paraissent dépourvus de sens, ainsi que l'opposition du réel et de l'idéal-possible. Si tout ce qui est rationnel est effectif, il s'ensuit en effet que le réel est ce qui doit être, le possible réel.

\section{IV.}

De cela, on peut tirer comme maxime de conduite que ce qui n'est pas effectif ne peut pas et ne doit pas servir d'étalon pour évaluer ce qui l'est, car ce qui n'est pas effectif n'est pas simplement possible, mais est pour Hegel tout à fait factice, et relève tout entier de la fantaisie. Ce procédé est impropre et repose sur une erreur qui consiste à ne pas reconnaître la rationalité du réel, à mépriser la réalité en la tenant pour insensée.

Mais cette attitude n'est pas simplement infondée, injustifiée. Elle est aussi dangereuse. Il y a d'abord un risque pour soi : celui de devenir hypocondriaque et de n'être 
jamais content. En effet, la critique du réel à la lumière de l'idéal apparaît comme figure du mauvais infini : la poursuite des idéaux mène à un mouvement sans fin, engendre une suite de transformations sans terme, puisque l'utopiste, l'idéaliste, n'est jamais satisfait, toujours désireux de conformer le monde à son idée et sans cesse en train de déterminer autrement cette idée. Autrement dit, la présentification de l'idéal demanderait un temps infini et ne serait jamais achevée. Plus exactement, il est impossible qu'elle le soit jamais, car ce qui est proprement idéal est impropre à la réalisation. En un sens, on peut dire qu'il est impossible. Il semble que l'idéal ne cesse de s'autodétruire, si bien qu'il ne pourra jamais être réel. Hegel dit bien dans l'addition du $§ 396$ que la réalisation de l'idéal est impossible. En effet, le jeune homme ou n'importe quel mécontent refuse de placer l'idéal dans la réalité — refus contre lequel s'élève Hegel : il suffit pour eux que quelque chose soit effectif pour qu'il perde son droit au titre d'idéal. Ainsi le jeune homme interprète-t-il systématiquement la réalisation de ce qui n'est pas idéal dans l'idéal, de ce qui est substantiel en lui, de l'universel, comme une trahison de son idéal, comme une « chute » hors de l'universel. En somme, ces idéalistes sont comme des enfants déçus de constater que ce qu'ils obtiennent est moins bien que ce qu'ils avaient rêvé. Mais pour Hegel s'impose comme conclusion non pas que le réel n'est pas à la hauteur de la pensée, mais que leur pensée est inadéquate au réel, que leur rêve est vain. Si l'idéal n'existe qu'en ne se réalisant pas, on comprend que l'idéaliste soit toujours insatisfait. Il est alors comme la belle âme de la Phénoménologie, qui, pour préserver la pureté de son intériorité, se maintient dans l'impuissance. Tous deux font trop les « dégoûtés » devant le fini, pour parvenir à la moindre effectivité (Hegel, Encyclopédie I 526 [Add. § 92]).

Mais que l'éternel mécontent se gâche la vie est une chose. C'en est une autre qu'il mette les autres en danger. Or, il semble bien que la fausse compréhension du possible et du réel fasse courir un tel risque. Pour Hegel, vouloir forcer le cours des événements contre la situation présente et les individus, vouloir de force faire entrer l'idéal dans les faits, conduit à 
des événements tels que la Terreur, qui voient une «volonté négative » (Hegel, Principes $\S 5 \mathrm{R})$ se traduire par une furie destructrice ${ }^{5}$. Il est à penser que si ces idéaux-là avaient été véritablement des possibles simplement privés d'existence, leur réalisation n'aurait pas provoqué une pure et simple destruction. Il n'y a pas de raison que le possible s'autodétruise, il y en a davantage que la fantaisie ou les produits de l'imagination subissent un tel sort. C'est pourquoi Hegel est très opposé à une conception poïétique de l'histoire, selon laquelle les événements et le cours des choses seraient la fabrication ex nihilo des individus, un pur produit du libre-arbitre. C'est pour lui une représentation de l'histoire destructrice, on pourrait même dire terroriste ${ }^{6}$.

\section{V.i}

On peut alors s'interroger sur le sens de ce qui se présente comme une identification du possible et du réel. En effet, refuser l'existence de possibles subsistants par soi et rationnels en dehors du réel revient à affirmer que le réel est la seule source de possibilité, ou que réalité et possibilité se rejoignent dans la rationalité. Il n'est pas illégitime de se demander si cette identification ne se fait pas au détriment du possible. Sa signification modale ne devient-elle pas purement formelle? Bien plus, affirmer que le devoir-être n'a pas de consistance, n'est-ce pas liquider le possible? N'est-ce pas reconnaître que seul le réel est, autrement dit, que rien d'autre ne peut être que ce qui est déjà? Serait alors supprimée toute alternative. Si ce qui est est ce qui doit être, s'il n'y a pas d'autrement, comment faire encore une place au possible?

N'est-ce pas finalement supprimer l'existence même de la modalité? En effet, il semble que la modalité existe du fait de sa pluralité. Le mode est ainsi nécessairement pluriel. Il doit être décliné, sans quoi on a affaire à une substance ou un attribut qui constitue l'essence permanente d'une substance. Parce que le mode est une détermination ou une 
déclinaison — de la relation objective ou de la qualité du jugement selon qu'on prenne la modalité au sens objectif ou qu'on la tienne pour la forme des jugements —, il semble impliquer une pluralité de modes possibles : traditionnellement, la possibilité et l'impossibilité, l'existence et l'inexistence, la nécessité et la contingence. Qu'une chose se fasse d'une certaine façon implique qu'en théorie, d'autres façons sont possibles. L'usage de la langue nous en donne une idée : s'interroger sur le mode dans lequel est écrit un morceau de musique (majeur, mineur) implique qu'il en est plus d'un, s'interroger sur les modalités de paiement signifie qu'en théorie, il y a plusieurs façons dont un paiement peut s'effectuer, etc.

S'il n'y a plus que la réalité qui seule existe, si le possible est évacué dans cette identification, alors la modalité elle-même peut sembler dissoute, d'autant que Hegel paraît identifier (par la suite) effectivité et nécessité. De ce réel, on ne dirait pas qu'il est la seule modalité, mais qu'il est au-delà de la modalité. À la limite, on ne dirait plus des choses qu'elles sont réelles (si elles ne peuvent être possibles), mais simplement qu'elles sont.

Si les détracteurs de Hegel se sont alarmés de ce qu'ils ont compris comme une disparition du possible et de la modalité, il semble que cette identification du possible au réel soit inquiétante au sein même du système hégélien. En effet, comme l'explique Bernard Mabille $^{7}$, pour sortir l'absolu du flou au sens de l'opacité, de l'indétermination, dans la Doctrine de l'essence, Hegel a besoin de la différenciation des catégories modales, pour éviter que l'absolu se résume dans l'unité indéterminée et immédiate de l'essence et de l'existence, de l'intérieur et de l'extérieur. En identifiant toutes les modalités de manière immobile, on prend le risque de perdre la détermination de l'absolu, de le priver d'effectivité et d'en faire une forme d'être pur.

Il apparaît donc que si l'aversion du réel inspirée par l'attachement aux idéaux représente, selon Hegel, un danger, le procès du devoir-être par la philosophie spéculative 
n'est pas non plus sans risque, puisqu'il pose d'une part une difficulté au sein du système hégélien lui-même et d'autre part un problème majeur relatif à ses implications.

\section{V.ii}

Cette critique du devoir-être a pu être comprise comme une éviction pure et simple de la possibilité. Or, quelles seraient les conséquences d'une telle suppression? N'est-ce pas réériger une forme de nécessitarisme? Faut-il comprendre l'attitude du philosophe de l'histoire qui critique les idéaux et celle de l'homme fait comme un geste de résignation, comme l'acceptation d'un destin, comme un renoncement à l'action face au poids du monde? N'est-ce pas un fatalisme et une justification de l'ordre établi? C'est ce qu'a pu défendre Nietzsche. Déjà dans la première Considération inactuelle, celui-ci dénonçait dans le hégélianisme un «culte du réel identifié au rationnel, c'est-à-dire la divinisation du succès ». Dans la deuxième Inactuelle, le hégélianisme était également interprété au premier chef comme une idolâtrie du réel et des faits considérés comme la sanction d'une nécessité immanente et anonyme où tout se justifie, où chaque événement vérifie le succès de la Logique ou de l'Idée, entraînant la soumission mécanique à tous les pouvoirs, caractérisation qui aboutit à la formule célèbre : «Pour Hegel, le sommet et le point final du processus mondial coïncident dans sa propre existence berlinoise » (Nietzsche 148-149). L'histoire suivrait un déroulement nécessaire entraînant une forme d'esclavage, toute résistance au mouvement de l'histoire devenant vaine. Les individus n'auraient plus qu'à acquiescer et à se faire les dociles instruments de ce qui a été décidé et de ce qui se réalise sans eux.

Ce fatalisme, issu de l'identification du possible au réel, serait perceptible pour Nietzsche dans la conception hégélienne des «grands hommes », qui auraient plus de chance que de génie. Il ne s'agirait pas de héros, d'individus d'élite qui ouvrent des perspectives nouvelles. Ils seraient plutôt «l'expression plus précise des lois de l'histoire, comme des 
bulles qui se forment à la surface des flots »(148-149), des individus dont les fins particulières ont simplement coïncidé avec ce qu'exigeait le cours du monde.

\section{VI.}

La lecture, dans la Doctrine de l'essence, du chapitre intitulé «L'effectivité » dans la section du même nom, peut nous préserver de conclusions si déprimantes, car celui-ci vient expliquer, non que le possible est une chimère, une vue de l'esprit, mais qu'il s'identifie à l'effectif. Or, la reconnaissance de cette intimité, d'une part, produit en retour une conception plus riche, plus haute, plus noble du possible - elle lui donne en somme des lettres de noblesse - et, d'autre part, conduit à y lire une affirmation de la contingence, point que nous ne pourrons développer ici mais qui a été abordé à notre sens avec profondeur par Juliette Simont et Bernard Mabille.

L'effectivité est pour Hegel l'unité de l'existence et de l'essence, ou de l'extérieur et de l'intérieur. Le développement de L'Être, première partie de la Logique objective, la dialectique de l'être-là, n'a pas suffi à comprendre vraiment l'effectivité, la relation de l'être et de l'essence. Au début de la Doctrine de l'essence se dessine le risque de concevoir l'essence comme un simple substrat et d'en déduire un dualisme de l'être et de l'essence, qui se trouve au fond des autres dualismes apparence/essence, fondement/existence, soi/phénomène, extérieur/intérieur. Le mouvement de la Doctrine de l'essence va consister à réduire ces dualismes ou verticalités — au sens de rapports transcendants excluant la possibilité d'inscrire leurs membres dans un même plan —, en dégageant l'unité réflexive de ces termes apparemment irréconciliables.

Le chapitre «L'effectivité », dans lequel on trouve une part importante de la théorie hégélienne de la modalité, va montrer que l'effectivité de l'absolu, c'est-à-dire l'unité réflexive de l'extérieur et de l'intérieur, de l'être et de l'essence, n'est pas d'emblée atteinte, 
et ne peut être atteinte immédiatement, mais requiert la différenciation de l'effectivité à travers les catégories modales pour permettre une détermination de l'absolu. Nous n'évoquerons ici que la première figure de l'effectivité.

Hegel fournit à la première figure («A. Contingence, ou effectivité, possibilité et nécessité formelles ») une caractérisation en deux temps :

(1) L'effectivité de l'absolu est d'abord formelle, parce qu'elle est d'abord immédiate. C'est d'abord l'existence ou l'être en général, une extériorité formelle. Être effectif, c'est simplement exister ou avoir une qualité, être quelque chose (mais dans le registre de l'essence). L'effectif a une certaine consistance, mais ne semble pas être la détermination d'une essence.

(2) Néanmoins, à la réflexion, comme effectivité, il s'agit d'une unité — bien que formelle (non réfléchie) — de l'intériorité et de l'extériorité, de l'essence et de l'être. Par conséquent, à ce moment, l'effectivité ne se réduit pas à l'existence immédiate, mais est aussi possibilité : «Ce qui est effectif est possible»(Hegel, Science I 248). L'effectif est ici compris à partir du possible. L'effectivité formelle se manifeste d'abord comme possibilité, parce qu'elle n'est pas juste être-là, extériorité pure, mais extériorité unie à l'intériorité de l'essence. L'effectif a une profondeur. Il relève de l'essence, mais quelle consistance a-t-il encore? Ne perd-il pas sa réalité en s'assimilant au possible?

Que l'effectif soit possible ne le déréalise pas, selon Hegel, mais au contraire, lui donne plus de réalité, puisque cela empêche de confondre l'effectif avec une existence simplement donnée, simplement rencontrée dans l'expérience. Nous voudrions ajouter que ce développement permet de donner plus de profondeur à la notion de possible, en empêchant de le confondre avec un moindre être ou l'absence de contradiction.

Comment se déploie la richesse de l'effectivité, comment se déploie l'unité du possible et de l'effectif? L'effectif formel se confond avec le possible, ou le possible est l'être 
en soi de l'effectif. Hegel rompt manifestement avec la conception kantienne du possible et du réel comme deux dimensions hétérogènes, ce que critiquera la Doctrine du concept. Est-ce pour revenir à une continuité dynamique entre les deux? L'exposition des deux moments du possible va infirmer cette hypothèse :

(1) Le possible est quelque chose de positif. C'est "l'être réfléchi dans soi-même », c'est-à-dire l'identité. Cette définition du possible correspond à son critère interne : ce qui n'est pas contradictoire est possible en dehors de toute réalisation.

(2) Mais le possible est aussi quelque chose de négatif : un être déficient qui est complété dans et par un autre, l'effectif. Le possible est ce qui tend à l'existence sans pourtant exister encore : il n'est pas simplement identique à soi, mais prétend à l'existence. Il a le statut d'une intention. Il tend vers son autre, son négatif.

Hegel va établir le caractère unilatéral et insuffisant de cette caractérisation du possible, pour indiquer qu'il faut penser le possible autrement pour concevoir vraiment sa relation à l'effectivité.

La possibilité est d'abord formellement identité à soi. En ce sens, « est possible tout ce qui ne se contredit pas » (250). C'est pour Hegel une des définitions données par Leibniz. Avec une partie de la tradition post-leibnizienne, il comprend l'absence de contradiction comme identité. Quelle valeur a cette conception du possible? Elle semble indiquer que tout, c'est-à-dire n'importe quoi, est possible, car tout semble identique à soi. On peut mettre n'importe quoi en équation avec lui-même. Cette notion du possible ne détermine rien. Elle n'a pour Hegel aucun intérêt, puisque dire, en ce sens, que quelque chose est possible, c'est comme ne « rien » dire (250). Cette conception du possible est donc stérile. Mais elle possède un autre défaut : comprise de la sorte, comme identité, la possibilité signifie son contraire, l'impossibilité. 
Dire que A est possible, c'est dire que A est A. Mais peut-on être ainsi soustrait tout à fait à la contradiction? Si chaque chose est déterminée, se définit par rapport à son autre et a donc sa négation en soi, alors rien n'est plus possible ou tout est impossible. En effet, tout contenu a des déterminations : il porte en lui la différence. C'est un grand thème hégélien qui est repris d'abord ici : celui de l'épreuve de la contradiction de toute identité. En tant que la possibilité n'est pas que forme, mais aussi contenu, elle s'écoule dans l'impossibilité. Si toute identité se révèle contradiction, alors le possible est aussi bien impossible.

On pourrait penser que la notion de possible est ici critiquée au moyen d'une idée générale et d'une façon assez extérieure qui n'implique pas vraiment la dimension de la modalité. Il semble que ce ne soit pas le cas. D'abord parce que la seconde caractérisation du possible fait aussi de celui-ci un impossible.

Hegel a dit que, dans son versant négatif, le positif est l'être déficient qui trouve son complément dans l'effectivité. Ce n'est pas simplement l'identité à soi, mais aussi «un moment de la totalité » (250-251). En ce sens, être possible c'est être « seulement ${ }^{8} »(251)$ possible, c'est là le devoir-être. La possibilité est alors un moment, essence incomplète posée, distinct de la forme absolue et qui attend d'être complété. C'est l'essence qui prétend à l'existence, et non pas seulement l'essence formelle. Mais ce devoir-être privé d'être n'a pas de vérité, il n'est pas en soi, ou plutôt il est un moment de la forme absolue, il a sa vérité dans un autre. La possibilité qui n'est que possibilité est donc encore contradiction ou impossibilité. En soi, le possible est déterminé à ne pas être, à être impossible.

Dans les deux cas, l'être du possible réside dans un être autre, dans une contradiction et donc se renverse en impossibilité. Mais Hegel va ajouter encore une raison plus profonde à l'écoulement de ces possibles dans l'impossibilité. Être possible signifie être seulement un contenu possible. Cela signifie qu'est possible un autre contenu que le contenu possible. La possibilité semble, en effet, consister dans le rapport de comparaison des deux contenus. 
Affirmer A, c'est donc aussi affirmer non-A (- A). Le A possible contient donc le non-A possible. Par conséquent, un possible contient sa propre impossibilité. Si je dis «il est possible que Pierre vienne », l'énoncé de la possibilité de la venue de Pierre implique la possibilité qu'il ne vienne pas, sans quoi sa venue serait réelle ou nécessaire. On a objecté à Hegel qu'il n'y a pas là impossibilité et contradiction, mais seulement contrariété : la proposition A contient la possibilité que non-A (la possibilité que Pierre ne vienne pas) et non sa propre impossibilité (il est impossible que A, que Pierre vienne). Il n'est pas sûr que l'objection porte, car, comme l'a montré B. Mabille (202-203) ${ }^{9}$, Hegel ne dit pas exactement que le possible contient sa contradiction, mais que la possibilité est contradiction, impossibilité.

La contradiction du possible est révélatrice du caractère impropre de cette double conception du possible : si le possible c'est cela, alors c'est quelque chose qui se détruit luimême, dont on ne peut rien dire, qui est pauvre et incohérent. Elle conduit pourtant à la vérité du possible. La contradiction dégagée à la fois signe l'échec des conceptions traditionnelles du possible et conditionne la compréhension vraie du possible. Comment, à partir de la contradiction et de sa structure contradictoire, le possible est-il compris par Hegel?

Comprendre que le possible contient son autre, aux yeux de Hegel, revient à saisir que la possibilité est la contradiction immédiate qui pourtant se subsume, c'est-à-dire que la possibilité « devient effectivité »(252).

On gardera présent à l'esprit que Hegel travaille sur l'idée que d'abord l'effectif est possible, c'est-à-dire sur l'union du possible et de l'effectif, et ce afin de donner une effectivité à l'absolu. La mise au jour de la structure contradictoire du possible implique que c'est l'unité du possible et de l'effectif qui est cette contradiction, contradiction qui n'est pas l'opposition de deux termes extérieurs étrangers l'un à l'autre pour Hegel, mais le renversement constant de l'un dans l'autre. Le passage est difficile, mais permet de 
déterminer «dans quelle mesure la possibilité est effectivité» (252). Certes, elle n'est pas encore toute effectivité. Mais la conclusion importante est que «[t]out possible a par conséquent en général un être ou une existence ». Cela signifie que le possible n'est pas un effectif imparfait, autrement dit que le rapport du possible à l'effectif n'est pas de l'ordre d'un passage, lors duquel il faudrait ajouter quelque chose au possible - le compléter pour en faire quelque chose d'effectif. L'idée d'un passage entre un non-étant que serait le possible et un étant que signifierait l'effectif est en effet problématique, et déjà Leibniz avait montré que le possible a un être, car il tend à être quelque chose (devoir-être). Cela signifie, par suite, que la considération abstraite du possible, la spéculation sur un possible séparé de l'effectif - ce à quoi se livraient le jeune homme et les hommes mécontents de l'état du monde — n'a pas de sens si le possible n'est rien hors de son unité avec l'effectif. Cela ne revient pas non plus à asseoir un nécessitarisme, à appuyer l'idée que tout ce qui est possible se réalise, puisque Hegel — ce que nous ne développerons pas ici — va expliquer, dans les lignes qui suivent, que l'unité contradictoire de l'intérieur et de l'extérieur, de l'essence et de l'existence, du possible et de l'effectif, est à saisir comme contingence. Le possible a un être : il est un élément d'une unité effective (un moment de la contingence de l'effectif). C'est une détermination de l'effectivité, qui de ce fait prend une consistance et se distingue du simple être là, et non un pré-effectif, un en deçà essentiel par rapport à l'effectivité (204-205).

Hegel a donc indiqué les limites de la compréhension classique du possible, qui fait de lui quelque chose qui, pour être non contradictoire, n'en est pas moins un moindre-être, séparé par un passage du réel, un ineffectif opposé à l'effectif, mais qu'on pourrait préférer à l'effectif et dont on pourrait user pour critiquer ce dernier. Si l'effectivité n'est pas quelque chose qui vient s'ajouter de l'extérieur au possible, s'il ne suffit pas de fournir au possible un complément pour qu'il se réalise, alors il faut critiquer, comme le fait Hegel, les entreprises de ceux qui prétendent travailler à la réalisation du possible de leur choix. Il y a bien l'idée, 
chez ces idéalistes, que leur idéal est simplement séparé du réel par un supplément d'être qui reste à trouver dans la mobilisation des énergies, etc. C'est penser que l'effectivité est, au regard du possible, quelque chose de contingent, ce en quoi, comme le montre ce chapitre de la Logique, ils se trompent.

\section{VII.}

Il y a pourtant, pour Hegel lui-même, une certaine vérité de cet idéalisme. Ces hommes partent d'une intuition qui n'est pas fausse, en présentant leurs idéaux comme ce qui doit être. Ce faisant, ils soupçonnent l'existence d'une continuité entre essence et existence, possible et réel, car ils affirment que leur idéal a des droits à l'existence. Aux yeux de Hegel, Leibniz a bien fait de voir dans le possible un devoir-être au-delà de la non-contradiction de l'identité, soit d'avoir indiqué que l'essence est animée d'une tendance à l'effectivité. Pourtant, il ne pense le possible que comme tension vers l'existence, comme puissance d'effectuation que seule l'intervention divine peut effectuer. De même, les idéalistes ne vont pas au bout de leur idée. Ils sentent que le vrai, le rationnel, doit être, autrement dit que le possible ne doit pas être simplement possible. Mais s'ils étaient tout à fait cohérents, ils admettraient que les droits à l'existence du rationnel sont reconnus, autrement dit que ce qui doit être a une effectivité, ou que le possible est effectif.

Signe qu'il y a une certaine vérité dans l'attitude de ces jeunes idéalistes et éternels mécontents, c'est qu'on voit Hegel lui-même présenter parfois le développement de l'esprit comme une possibilité qui s'actualise. Pourtant, on ne se méprendra pas sur le sens de cette idée qu'on rencontre, par exemple, dans l'Introduction des Leçons sur la philosophie de

\section{l'histoire :}

Ici il n'y a qu'à indiquer que l'esprit commence par son infinie possibilité, mais seulement sa possibilité, qui renferme son contenu absolu comme en soi, comme la fin et le but qu'il n'atteindra que dans son résultat, qui est alors simplement sa réalité. 
Dans l'existence, la succession apparaît ainsi comme un progrès de l'imparfait vers le plus parfait et ce n'est pas abstraitement seulement que l'imparfait doit être conçu comme tel, mais comme un facteur qui contient aussi en soi le contraire de soi-même, comme germe, comme instinct. La possibilité indique tout au moins par réflexion quelque chose qui doit réellement se réaliser, et la dynamis d'Aristote est exactement potentia, force et pouvoir. L'imparfait, en tant que le contraire de soi en soi, est la contradiction qui sans doute existe mais est tout aussi bien supprimée et résolue, l'instinct, l'impulsion de la vie spirituelle en soi pour briser l'écorce de la nature, des sens, des éléments étrangers et pour parvenir à la lumière de la conscience, c'est-àdire à soi-même (Hegel, Leçons 52).

Nous retiendrons ici que Hegel présente le possible comme effectif, en disant que la possibilité doit se réaliser. Tout le possible est destiné à se réaliser. Il n’y a pas l'idée de possibles laissés inexploités. Le possible a toujours la puissance de se réaliser; autrement dit, il n’y a pas pour l'esprit de possible qui resterait simplement en puissance : la possibilité de ne pas passer à l'acte est écartée. Alors, le cas d'une puissance qui ne passerait pas à l'acte est écarté. L'idée est très difficile, car il s'agit de nier l'existence de possible seulement possible, puissances absolument sans acte, sans pour autant reconduire la définition destinale du possible des Mégariques, selon laquelle le possible est ce qui est ou sera, définition qui en annule la consistance.

Pour la comprendre, il faut souligner que le caractère effectif du possible n'implique pas du tout que le réel soit immédiatement tel qu'il doit être. L'effectivité du possible — qui est une manière de contrer son impuissance dans les autres conceptions - s'accommode d'un échelonnement temporel du plein accomplissement de cette effectivité. Le concept de l'esprit est ainsi toujours déjà effectif, mais ne sera pleinement effectif que dans le résultat de son développement.

\section{VIII.}

Que doit saisir le jeune homme pour devenir un homme et que doivent comprendre ceux qui se plaignent du monde? Hegel l'exprime en deux formules dans l'Addition au $§ 396$ 
de l'Encyclopédie et dans l'Introduction des Leçons : ils doivent apercevoir que « le monde comme un monde subsistant-par-soi, [est] pour l'essentiel tout achevé » et que «que le monde réel est tel qu'il doit être, que le vrai bien, la raison divine universelle est aussi la puissance propre de se réaliser. » Ils doivent apprendre à vouloir ce qui est et non à courir après des idéaux. La reconnaissance de cette identité du possible et de l'effectif implique que la volonté véritable veut pour et non contre, qu'elle a un intérêt pour et non contre le monde (Hegel, Encyclopédie III 432 [Add. § 396]), car se dresser contre ce qui est précipite dans le ressentiment. Ce n'est pas pour autant une défaite de leur désir face au poids des choses, défaite qui rendrait vaine toute initiative personnelle. En effet, que le possible soit effectif n'implique pas que le monde soit d'emblée tel qu'il doit être, qu'il n'y ait plus jamais rien de nouveau sous le soleil. Si, pour Hegel, on ne peut pas transformer le réel en le traitant comme de la pâte à modeler, si on ne peut pas faire advenir des idéaux, il reste à imprimer sa marque sur l'effectivité en saisissant les possibilités du temps. En effet, le concept ne se déploie pas comme un programme. Certes, l'homme ne produit donc que ce qui en un sens est déjà là, dit Hegel $(438)^{10}$. Mais il s'agit là d'une préexistence logique (et non chronologique) de tout ce qui se déploie. Ce n'est donc pas parce que quelque chose est déjà là dans le réel, qu'il n'y aurait plus rien à faire, et Hegel parle bien à propos de l'homme fait d'un progrès opéré grâce à son activité pratique.

IX.

Le grand homme est alors précisément celui qui prend acte pleinement de l'identité du possible et de l'effectif, ou du cercle qui existe entre l'être et la volonté. Il cherche «le nécessaire », «ce qui appartient aux possibilités du temps », ce qui est défini et susceptible d'accomplissement : 
De tels individus n'avaient pas en ce qui concerne leurs fins, conscience en général de l'Idée; mais ils étaient des hommes pratiques et politiques. C'étaient aussi des gens qui pensaient et qui savaient ce qui est nécessaire, et ce dont le moment est venu. C'est à savoir la vérité de leur temps et de leur monde, pour ainsi parler, la race prochaine qui existait déjà intérieurement. C'était leur affaire de connaître cette valeur générale, l'échelon nécessaire, prochain, de leur univers, d'en faire leur fin, d'y consacrer leur énergie. C'est pourquoi les hommes de l'histoire universelle, les héros d'une époque, doivent être reconnus comme les sages; leurs actes, leurs discours sont ce qu'il y a de mieux à leur époque. [...] Ce sont de grands hommes parce qu'ils ont voulu et accompli une grande chose, non imaginaire et présumée, mais bien juste et nécessaire (Hegel, Leçons 35-36).

Les grands hommes se présentent alors comme des vouloirs singuliers qui viennent médiatiser empiriquement l'avancée de l'Esprit du monde, et ce en saisissant ce pour quoi « les temps sont venus ». C'est pourquoi Hegel peut dire de César devenant le seul maître à Rome que l'événement ne signifie pas simplement un gain particulier, mais qu'il répond à « un instinct qui accomplit ce qu'en soi le temps réclamait », c'est-à-dire qu'il correspond à une détermination nécessaire dans l'histoire de Rome et du monde (35).

\section{X.i}

Hegel nous enseigne ainsi que l'objet de la volonté n'est pas le devoir-être mais l'être. D'un point de vue historique, il affirme que le grand homme n'est pas celui qui recherche un idéal, mais celui qui comprend ce qui est nécessaire, ce qui est appelé par la réalité, celui qui veut non l'utopie mais le substantiel. Il devient donc stérile d'opposer au présent une représentation de fantaisie et de tenter de s'en approcher. Hegel ne cesse de critiquer le devoir-être. Il n'y a pas ce qui doit être, dit-il, mais il y a ce qui est. Autrement dit, ce qui est est ce qui doit être, l'absolu et l'effectif. C'est pourquoi, dans la Préface des Principes, il souligne que la philosophie doit se garder d'être édifiante, d'opposer un idéal à l'effectif, car ce qui est rationnel est effectif et ce qui est effectif est rationnel : « la philosophie, parce qu'elle est l'examen-approfondi du rationnel, est par là même l'appréhension du présent et 
de l'effectif, [et] non pas l'établissement d'un au-delà qui devrait être Dieu sait où — ou dont on sait bien dire en fait où il est, à savoir dans l'erreur de la pensée ergoteuse vide, unilatérale »(Hegel, Principes 103). Le jeune homme et le mécontent doivent apprendre à vouloir ce qui est déjà là, même s'ils commencent par développer une résistance face à cette idée. Cette résistance est d'ailleurs inscrite dans le réel : avoir des idéaux fait partie du réel. Ce que le jeune homme ne sait pas, c'est que son aspiration fait partie de ce qui est, qu'il s'agit d'un moment de la volonté et que la défaite de ce moment est inscrite dans le réel ${ }^{11}$. En ce sens, avoir des idéaux fait partie du réel, est possible. Avoir un idéal — ce que le jeune homme ne comprend pas — fait partie de ce qui est. C'est un des moments de la volonté.

\section{X.ii}

L’argument généralement mobilisé contre la pensée hégélienne de la modalité vise l'identité — dont à notre sens le caractère dialectique n'est pas compris — des différentes catégories de la modalité. L'affirmation de leur identité est interprétée par les détracteurs de la logique hégélienne comme impliquant la suppression de leurs caractères respectifs spécifiques. Mais cette perte aurait toujours un bénéficiaire, l'identification se ferait au profit d'une des catégories modales, tantôt l'une tantôt l'autre, selon l'orientation des critiques. La suppression des logiques modales induirait alors le renforcement de l'une d'entre elles, soit une dissolution de la modalité au profit d'un mode. Concernant les catégories du possible et du réel, le sacrifice de leurs caractères propres a été symétriquement attaqué. On a alors pu dénoncer le fait que leur identification chez Hegel se faisait aussi bien au détriment du possible et à la faveur du réel, qu'au détriment du réel et à la faveur du possible. Il a ainsi été reproché à Hegel d'identifier le possible à l'effectif et d'engendrer un monde dont la nécessité paralyse la volonté, un monde sans possibilité mais dominé par la réalité du réel, voire la logique du fait accompli. C'est l'objection que nous avons présentée. Mais le 
reproche inverse lui a été adressé, celui d'avoir identifié l'effectif au possible et par là d'avoir détruit ce que l'existence a de concret. Kierkegaard a ainsi refusé la manière dont Hegel repense la modalité — possibilité et réalité ne sont pas exclusives l'une de l'autre —, s'en prenant plus précisément au sort réservé par lui à la réalité dans cette refonte des catégories modales. Kierkegaard défend la distinction nette entre ce qui est possible, c'est-à-dire concevable, et ce qui est existant, qui implique que quelque chose soit donné dans l'expérience, et refuse ainsi le court-circuit hégélien :

Pour l'existant, d'exister est le suprême intérêt, et l'intérêt à l'existence est la réalité. Ce qu'est la réalité ne se laisse pas exprimer dans le langage de l'abstraction. La réalité est un intêressetre l'unité abstraite hypothétique de la pensée et l'être. L'abstraction traite de la possibilité et de la réalité, mais sa conception de la réalité est une fausse interprétation, car le plan sur lequel nous sommes n'est pas celui de la réalité, mais celui de la possibilité. L'abstraction ne peut se rendre maîtresse de la réalité qu'en l'abolissant, mais l'abolir signifie justement la transformer en possibilité. Tout ce qui est dit de la réalité dans le langage de l'abstraction et à l'intérieur de l'abstraction est dit à l'intérieur de la possibilité; dans le langage de la réalité toute l'abstraction se rapporte en effet comme une possibilité à la réalité et non pas à une réalité qui se trouverait à l'intérieur de l'abstraction et de la possibilité. [...] Tout savoir sur la réalité est possibilité; la seule réalité dont un être existant ne se borne pas à avoir une connaissance abstraite est la sienne propre, qu'il existe; et cette réalité constitue son intérêt absolu. L'exigence de l'abstraction à son égard est qu'il se désintéresse pour qu'il puisse savoir quelque chose; l'exigence de l'éthique, qu'il s'intéresse infiniment à l'existence (Kierkegaard 267-268).

Quand d'autres avaient dénoncé la dissolution d'un possible identifié au réel, Kierkegaard se dresse contre la déréalisation d'un réel identifié au possible - le concept. Ce réel, oppose-t-il à Hegel, est une abstraction : il n'a plus rien de commun avec le réel que nous connaissons et que nous sommes, avec celui qui nous est familier et dont nous faisons l'expérience. En soutenant que l'effectif est possible, Hegel perdrait le sens de l'existence, s'éloignerait du réel. En «possibilisant» le réel, il tenterait d'étendre les voies de la logique là où elles ne peuvent avoir cours, là où domine le mouvement de l'existence et des singularités. 
Notre argumentaire visait au contraire à pointer ceci qu'en disant que l'effectif est possible et que le possible est effectif, Hegel ne les détruit pas en leur ôtant leurs déterminations propres, mais leur assure une plus grande consistance. En étant possible, l'effectif cesse de se confondre avec la réalité comprise comme simple existence ou simple phénomène, comme simple il y a. Parce qu'il est possible, le réel n'est justement pas factualité, être-là, fait accompli, etc. En étant effectif, le possible cesse quant à lui de signifier un moindre-être, un en-deçà, un simplement ou seulement possible, puisqu'il est alors inscrit dans l'être, dans le monde : il possède un acte et une substance et n'a plus rien d'un doux rêve ou d'une spéculation aveugle détachée du souci de la réalité.

\section{Notes}

${ }^{1}$ Nous reprenons la traduction de Bernard Bourgeois. Hegel distingue en particulier l'âge du «Kind» (l'enfant), celui du « Jüngling » (le jeune homme), celui du «Mann» (l'homme fait) et celui du « Greis » (le vieillard).

${ }^{2}$ Platon, déjà, dans la République déplorait l'attitude de ces hommes toujours occupés à rêver des souffrances imaginaires, qui, ayant perdu toute aptitude pour les arts et pour les sciences, étaient devenus incapables de comprendre et de méditer.

${ }^{3}$ De même dans la Préface des Principes de la philosophie du droit, Hegel présente toute conscience subjective qui tient le présent pour quelque chose de vain, le toise de haut et prétend en savoir plus long, comme la vacuité même.

${ }^{4}$ Dans la Préface des Principes de la philosophie du droit, il évoque en effet cette mode qui voit chacun s'autoriser à expliquer ce que l'État, le gouvernement ou la constitution doit être. Hegel distingue sa démarche de cette attitude : «Ainsi donc, ce traité, en tant qu'il contient la science de l'État, ne doit être rien d'autre que la tentative de conceptualiser et d'exposer l'État comme quelque chose de rationnel au-dedans de soi. En tant qu'écrit philosophique, il faut qu'il soit au plus haut point éloigné de devoir construire un État tel qu'il doit être; l'enseignement qui peut résider en lui ne peut tendre à enseigner à l'État comment il doit être, mais plutôt comment cet État, l'univers éthique, doit être connu » (Hegel, Principes 105).

5 «La liberté universelle ne peut donc produire ni une œuvre positive ni une opération positive; il ne lui reste que l'opération négative; elle est seulement la furie de la destruction » (Hegel, Phénoménologie II 135). 
${ }^{6}$ L'histoire est néanmoins « faisable » par les hommes qui peuvent en modifier le cours, car c'est à leurs actions que revient l'actualisation de la rationalité du monde. Pourtant, si les hommes et les peuples font l'histoire c'est dans une situation donnée qui oriente ce qui est faisable ( $c f$. Bouton).

${ }^{7}$ Ce développement s'inspire largement de l'analyse conduite par Bernard Mabille dans la section 6 ( «L'effectivité de la contingence. Onto-logique du contingent») de son livre Hegel, l'épreuve de la contingence (177-211).

8 «[I]l a par conséquent la seconde détermination [qui consiste] à être seulement quelque chose de possible » (Hegel, Science I 251).

${ }^{9}$ Mabille répond ici à l'objet qui fait fond sur le fait que Hegel confondrait contraire et contradictoire.

10 «[L]'homme [fait] ne produit que cela même qui est déjà là » (Hegel, Encyclopédie III 432 [Add. § 396]).

${ }^{11}$ La succession d'une jeunesse idéaliste et d'un âge de la maturité réaliste résume d'ailleurs le parcours intellectuel de Hegel : "Cette progression vers le réalisme s'opère lentement et difficilement, dans cette crise dont Hegel a dit qu'elle marquait le laborieux passage de l'adolescence idéaliste au réalisme de l'âge adulte » (Bourgeois 59). Cet âge adulte viendrait alors avec la période de Francfort, celle de Berne étant encore teintée d'idéalisme.

\section{Ouvrages cités}

Bourgeois, Bernard, La pensée politique de Hegel, Paris, P.U.F., coll. Questions, 1992.

Bouton, Christophe, Le procès de l'Histoire. Fondements et postérité de l'idéalisme historique de Hegel, Paris, Vrin, coll. Bibliothèque d'histoire de la philosophie, 2004.

Hegel, G. W. F., Encyclopédie des sciences philosophiques, I : La science de la logique, trad. B. Bourgeois, Paris, Vrin, coll. Bibliothèque des textes philosophiques, 1970.

—, Encyclopédie des sciences philosophiques, III : Philosophie de l'esprit, trad. B. Bourgeois, Paris, Vrin, coll. Bibliothèque des textes philosophiques, 1988.

—, Leçons sur la philosophie de l'histoire, trad. J. Gibelin, Paris, Vrin, coll. Bibliothèque des textes philosophiques, 1998.

—, Phénoménologie de l'Esprit, t. II, trad. J. Hyppolite, Paris, Aubier, coll. Bibliothèque philosophique, 1941.

—, Principes de la philosophie du droit, trad. J.-F. Kervégan, Paris, P.U.F., coll. Quadrige, 2003. 
-, Science de la logique, t. I, livre 2: La doctrine de l'essence, trad., prés. et notes P.-J. Labarrière et G. Jarczyk, Paris, Aubier, coll. Bibliothèque philosophique, 1976.

Kierkegaard, Soren, Post-scriptum aux miettes philosophiques, trad. P. Petit, Paris, Gallimard, coll. Tel, 1989.

Mabille, Bernard, Hegel, l'épreuve de la contingence, Paris, Aubier, coll. Philosophie, 1999.

Nietzsche, Friedrich, Seconde Considération intempestive, trad. H. Albert, Paris, Flammarion, coll. GF, 1988.

Simont, Juliette, Essai sur la quantité, la qualité, la relation chez Kant, Hegel, Deleuze. Les «fleurs noires » de la logique philosophique, Paris, L'Harmattan, 1997.

—, «Les prédateurs du crépuscule dialectique (à propos du commentaire de la Science de la logique)», dans : P. Verstraeten (dir.), Hegel aujourd'hui, Annales de l'Institut de philosophie et de sciences morales (Université de Bruxelles), Paris, Vrin, 1995, p. 167-196. 